\title{
Classification of stable solutions for non-homogeneous higher-order elliptic PDEs
}

Abdellaziz Harrabi ${ }^{1}$, Belgacem Rahal ${ }^{2 *}$ (D) and Mohamed Karim Hamdani²

\section{"Correspondence:}

rahhalbelgacem@gmail.com

${ }^{2}$ Faculté des Sciences, Département de Mathématiques, Université de

Sfax, B.P. 1171, 3000, Sfax, Tunisia Full list of author information is available at the end of the article

\begin{abstract}
Under some assumptions on the nonlinearity $f$, we will study the nonexistence of nontrivial stable solutions or solutions which are stable outside a compact set of $\mathbb{R}^{n}$ for the following semilinear higher-order problem:

$$
(-\Delta)^{k} u=f(u) \text { in } \mathbb{R}^{n}
$$

with $k=1,2,3,4$. The main methods used are the integral estimates and the Pohozaev identity. Many classes of nonlinearity will be considered; even the sign-changing nonlinearity, which has an adequate subcritical growth at zero as for example $f(u)=-m u+\lambda|u|^{\theta-1} u-\mu|u|^{p-1} u$, where $m \geq 0, \lambda>0, \mu>0, p, \theta>1$. More precisely, we shall revise the nonexistence theorem of Berestycki and Lions (Arch. Ration. Mech. Anal. 82:313-345, 1983) in the class of smooth finite Morse index solutions as the well known work of Bahri and Lions (Commun. Pure Appl. Math. 45:1205-1215, 1992). Also, the case when $f(u) u$ is a nonnegative function will be studied under a large subcritical growth assumption at zero, for example $f(u)=|u|^{\theta-1} u\left(1+|u|^{9}\right)$ or $f(u)=|u|^{\theta-1} u e^{|u|^{q}}$, $\theta>1$ and $q>0$. Extensions to solutions which are merely stable are discussed in the case of supercritical growth with $k=1$.
\end{abstract}

PACS Codes: Primary 35J55; 35J65; secondary 35B33; 35B65

Keywords: higher-order equation; Liouville theorems; finite Morse index; Pohozaev identity

\section{Introduction}

This paper is devoted to the study of solutions, possibly unbounded and sign-changing, of the semilinear partial differential equation,

$$
(-\Delta)^{k} u=f(u) \quad \text { in } \mathbb{R}^{n}
$$

where $k=1,2,3,4, n \geq 1$ and $f \in C^{1}(\mathbb{R})$. Under some assumptions on the nonlinearity $f$, we will show that this problem does not possess a nontrivial solution with finite Morse index.

In the last decades, problems related to the nonexistence of finite Morse index solutions for second-, fourth- and sixth-order Lane-Emden equation on unbounded domains of $\mathbb{R}^{n}$ have received a lot of attention (see [2-12]).

(c) The Author(s) 2017. This article is distributed under the terms of the Creative Commons Attribution 4.0 International License (http://creativecommons.org/licenses/by/4.0/), which permits unrestricted use, distribution, and reproduction in any medium, provided you give appropriate credit to the original author(s) and the source, provide a link to the Creative Commons license, and indicate if changes were made. 
We now list some known results. We start with the second-order Lane-Emden equation

$$
-\Delta u=|u|^{p-1} u, \quad \text { in } \mathbb{R}^{n}, p>1,
$$

Farina [6] proved that nontrivial finite Morse index solutions of (1.2) exist if and only if $p \geq p_{c}(n)$ and $n \geq 11$, or $p=\frac{n+2}{n-2}$ and $n \geq 3$, where $p_{c}(n)$ is the so-called Joseph-Lundgren exponent. Also, in [13] several Liouville-type theorems are presented for stable solutions, where $f>0$ is a general convex, nondecreasing function. Extensions to solutions which are merely stable outside a compact set are discussed.

For the fourth-order Lane-Emden problem

$$
\Delta^{2} u=|u|^{p-1} u, \quad \text { in } \mathbb{R}^{n}, p>1,
$$

the subcritical case has been studied by Ramos and Rodriguez for finite Morse index signchanging solutions (see [14]). The supercritical case is more complicated and there are several new approaches dealing with (1.3). The first approach is to use the test function $v=-\Delta u$. To this end, one has to use Souplet's inequality [15], this will give an exponent $\frac{n}{n-8}+\epsilon_{n}$ for some $\epsilon_{n}>0$; see [16]. These results were improved in [12] by adapting Farina's approach with the restriction on the power $q<\frac{2}{3}$. The second approach was obtained by Cowan and Ghoussoub [3], Dupaigne et al. [17] and further exploited by Hajlaoui, Ye and one of the authors [7]. This approach improves the first upper bound $\frac{n}{n-8}+\epsilon_{n}$, but it again fails to catch the fourth-order Joseph-Lundgren exponent computed by Gazzola and Grunau [18]. It should be remarked that by combining these two approaches one can show that stable positive solutions to (1.3) do not exist when $n \leq 12$ and $p>1$; see [7]. Finally in [5], Dávila et al. employed a monotonicity formula-based approach and gave a complete classification of stable and finite Morse index (positive or sign-changing) solutions to (1.3). A remarkable outcome of this third approach is that it gives the optimal exponent. The main tool of [5] is a monotonicity formula, used to perform a blow-down analysis and reduce the nonexistence of nontrivial entire solutions for the problem (1.3), to that of nontrivial homogeneous solutions.

Thanks to the Liouville-type theorem with finite Morse index in [8], the authors proved the nonexistence result of sign-changing solutions for the sixth-order problem

$$
-\Delta^{3} u=|u|^{p-1} u, \quad \text { in } \mathbb{R}^{n}, p>1
$$

Let us give a brief sketch of their method. They proved various classification theorems and Liouville-type results for $C^{6}$-solutions belonging to one of the following classes: stable solutions, solutions which are stable outside a compact set of $\mathbb{R}^{n}$. These results apply to the subcritical case using the Pohozaev identity. In the supercritical case, motivated by the monotonicity formula established in [19], they reduced the nonexistence of nontrivial entire solutions for the problem (1.4), to that of nontrivial homogeneous solutions. Through this approach, they gave a complete classification of stable solutions and those finite Morse indices, whether positive or sign-changing. Also, this analysis reveals the existence of a new critical exponent called the sixth-order Joseph-Lundgren exponent, also they gave the explicit value of this exponent.

In this work, we are concerned with Liouville-type theorems for the nonlinear elliptic equation (1.1) for $k=1,2,3,4$. We prove Liouville-type theorems for solutions (whether 
positive or sign-changing) belonging to one of the following classes: stable solutions and solutions which are stable outside a compact set. Our proof is based on a combination of the integral estimates and the Pohozaev-type identity.

The paper is organized as follows. In Section 2 we state our main results, which are then proved in Section 4. Section 3 contains some important auxiliary tools, which are used in the proofs of the main theorems.

\section{Statement of the main results}

In order to state our results, we present first some assumptions on the nonlinearity $f$ :

$H_{1}$ : There exists a constant $\theta>1$ such that

$$
f^{\prime}(s) s^{2}-\theta f(s) s \geq 0, \quad \forall s \in \mathbb{R}
$$

$H_{2}$ : There exist constants $s_{0}>0, \theta>1$ and $C_{0}>0$ such that

$$
C_{0}|s|^{\theta+1} \leq f(s) s, \quad \forall|s| \leq s_{0}
$$

$H_{3}$ : There exists a constant $0<\alpha_{0}<1$ such that

$$
\frac{2 n}{n-2 k} F(s)-\left(1+\alpha_{0}\right) f(s) s \geq 0, \quad \forall s \in \mathbb{R},
$$

where $F(s)=\int_{0}^{s} f(t) d t$.

Remark 2.1 (1) $H_{1}$ implies $H_{1}^{\prime}$ : There exist constants $s_{0}>0, \theta>1$ and $C_{0}>0$ such that

$$
C_{0}|s|^{\theta+1} \leq f(s) s, \quad \forall|s| \geq s_{0}
$$

Indeed, by $H_{1}$, we have $\frac{f}{|s|^{\theta}}$ is nondecreasing function for all $|s| \geq s_{0}$. This implies that

$$
C_{0}|s|^{\theta+1} \leq f(s) s, \quad \forall|s| \geq s_{0} .
$$

(2) $H_{1}$ implies the Ambrosetti-Rabinowitz condition (A-R): there exist constants $\widetilde{\theta}>2$ and $s_{0}>0$ such that

$$
f(s) s \geq \tilde{\theta} F(s)>0, \quad \text { for }|s|>s_{0}
$$

Examples We easily verify that the following functions satisfy $H_{1}$ and $H_{2}$.

1. $f(s)=C_{0}\left(1+|s|^{q}\right)|s|^{\theta-1} s, \theta>1, q>0$ and $C_{0}>0$.

2. $f(s)=|s|^{\theta-1} s e^{|s|^{q}}, \theta>1$ and $q>1$.

3. $f(s)=\sum_{i=1}^{i=i_{0}} c_{i}|s|^{\theta_{i}-1} s$, with $\theta_{i}>1 \forall i=1,2, \ldots, i_{0}$ and $c_{i}>0 \forall i=1,2, \ldots, i_{0}$. In this example we choose $\theta=\min _{1 \leq i \leq i_{0}}\left(\theta_{i}\right)$.

The examples (1) and (2) show that $f$ can have an exponential growth at infinity. Therefore, clearly an adequate behavior of $f$ at zero is needed to obtain the Liouville theorem. The unique and important nonexistence result for stable solutions of the nonhomogeneous second-order equation (1.1) has been recently obtained in [13]. It is shown 
there, among other things, that (1.1) does not admit nontrivial stable or stable outside a compact set solution provided that $f$ is regular, positive, nondecreasing and convex function in $(0,+\infty)$. More precisely, under a mere nonnegativity assumption on the nonlinearity, the authors begin this work by stating that up to space dimension $n=4$, bounded stable solutions of (1.1) are trivial. For the next series of results, they restricted themselves to the following class of nonlinearities:

$$
f \in C^{0}\left(\mathbb{R}_{+}\right) \cap C^{2}\left(\mathbb{R}_{+}^{*}\right), \quad f>0 \text { is nondecreasing and convex in } \mathbb{R}_{+}^{*} .
$$

In order to relate the nonlinearity $f$ and the below exponents (2.3) and (2.4), they introduced a quantity $q$ defined for $u \in \mathbb{R}_{+}^{*}$ by $q(u)=\frac{f^{\prime 2}}{\bar{f}^{\prime \prime}}(u)$, whenever $f^{\prime \prime}(u) \neq 0$ and $q(u)=+\infty$ otherwise. They assumed that $q(u)$ converges as $u \rightarrow 0^{+}$and denote its limit by

$$
q_{0}=\lim _{u \rightarrow 0^{+}} q(u) \in \overline{\mathbb{R}}
$$

Define now $p_{0} \in \overline{\mathbb{R}}$ the conjugate exponent of $q_{0}$, by $\frac{1}{p_{0}}+\frac{1}{q_{0}}=1$. The exponent $p_{0}$ must be understood as a measure of the 'flatness' of $f$ at 0 . However, we establish their following theorem.

Theorem A [13] Assume that $f$ satisfies (2.1) and (2.2). Assume that $u \in C^{2}\left(\mathbb{R}^{n}\right)$ is stable solution of (1.1) with $k=1$. Then $u \equiv 0$ if any one of the following conditions holds:

1. $1 \leq n \leq 9$ and $1<\underline{p_{\infty}}$,

2. $n=10, p_{0}<+\infty$ and $1<p_{\infty}$,

3. $n \geq 11, p_{0}<p_{c}(n)$ and $1<p_{\infty}<p_{c}(n)$, where $\underline{p_{\infty}} \in \overline{\mathbb{R}}$ be defined by $\overline{q_{\infty}}=\lim \sup _{u \rightarrow+\infty} q(u), \frac{1}{\underline{p_{\infty}}}+\frac{1}{q_{\infty}}=1$.

A typical example of nonlinearity function $f$ satisfying the above conditions (2.1) and (2.2) is $f(u)=|u|^{\theta-1} u+|u|^{p-1} u$, where $p \geq \theta$. A simple calculation, we get $p_{0}=\theta$ and $\underline{p_{\infty}}=p$. We use this nonlinearity function to establish some new Liouville-type theorems. Our method is different from (and complementary to) the one used in [13]. It exploits the attractive character of the difference between $f^{\prime}(u) u^{2}-\theta f(u) u \geq 0$, if $p \geq \theta$, that is, $f$ satisfies $H_{1}$ and $H_{2}$. It will be shown in Theorem 2.1 that problem (1.1) does not possess nontrivial stable solutions if and only if $1<\theta<p_{c}(n), \forall p \geq \theta$. Also, we may consider nonlinearities with exponential growth at infinity, i.e. $p_{\infty}=\infty$ satisfying $H_{1}$ and $H_{2}$, as for example $f(u)=|u|^{\theta-1} u e^{|u|^{q}}, \theta>1$ and $q>0$; therefore, in view again of Theorem 2.1, one has $u \equiv 0$. Furthermore, the present paper is motivated by the interesting work [1], we shall revise the nonexistence theorem of Berestycki and Lions [1] if one substitutes their assumption, which is

$$
\int_{\mathbb{R}^{n}}|\nabla u|^{2}+\int_{\mathbb{R}^{n}} f(u) u<+\infty,
$$

by assuming that $u$ is stable or stable outside a compact set. Therefore sign-changing nonlinearities will also be considered and we do not require that $f^{\prime}(0)=0$ as the instructive example given by Berestycki and Lions [1] is $f(u)=-m u+\lambda|u|^{\theta-1} u-\mu|u|^{p-1} u$, where $\lambda, \mu$ are positive constants, $m \geq 0$ and $1<\theta, p$. Observe that the above nonlinearity satisfies $\left(H_{1}\right)$, thus we shall prove that equation (1.1) does not possess a nontrivial stable solution 
provided $1<p \leq \frac{n+2 k}{n-2 k}$ and $p<\theta$, also if $u$ is bounded solution to (1.1) and $m>0$, then $u \equiv 0$, for any $\theta \geq p$. If $p \leq \frac{n+2 k}{n-2 k} \leq \theta$ and $m>0$, it follows from the Pohozaev identity that there cannot exist a nontrivial solution of (1.1) which is stable outside a compact set. This result is similar to [1] for $k=1$. To conclude, this work completes the study of Dupaigne and Farina [13] since here we do not assume that $f$ is positive and convex function. Therefore, to be more concrete in our analysis of nonexistence, we will distinguish between stable and stable outside a compact set. We provide some elliptic decay estimates that we use frequently later in the proofs. Deriving the right decay estimates for solutions of (1.1) plays a fundamental role in most our proofs. On the other hand, we shall also consider the question of the nonexistence of stable solutions (positive or sign-changing) in the supercritical case of a second-order equation.

In order to state our results we need to recall the following.

Definition 2.1 A solution $u$ of (1.1) belonging to $C^{2 k}\left(\mathbb{R}^{n}\right)$

- is said to be stable if

$$
Q_{u}(\psi):=\int_{\mathbb{R}^{n}}\left|D^{k} \psi\right|^{2} d x-\int_{\mathbb{R}^{n}} f^{\prime}(u) \psi^{2} d x \geq 0, \quad \forall \psi \in C_{c}^{k}\left(\mathbb{R}^{n}\right),
$$

where

$$
D^{k}= \begin{cases}\Delta^{\frac{k}{2}} & \text { for } k=2,4 \\ \nabla \Delta^{\frac{k-1}{2}} & \text { for } k=1,3\end{cases}
$$

- is stable outside a compact set $\mathcal{K} \subset \mathbb{R}^{n}$, if $Q_{u}(\psi) \geq 0$ for any $\psi \in C_{c}^{k}\left(\mathbb{R}^{n} \backslash \mathcal{K}\right)$.

More generally, the Morse index of a solution is defined as the maximal dimension of all subspaces $E$ of $C_{c}^{k}\left(\mathbb{R}^{n}\right)$ such that $Q_{u}(\psi)<0$ in $E \backslash\{0\}$. Clearly, a solution is stable if and only if its Morse index is equal to zero.

Remark 2.2 It is well known that any finite Morse index solution $u$ is stable outside a compact set $\mathcal{K} \subset \mathbb{R}^{n}$. Indeed, there exist $K \geq 1$ and $X_{K}:=\operatorname{Span}\left\{\phi_{1}, \ldots, \phi_{K}\right\} \subset C_{c}^{k}\left(\mathbb{R}^{n}\right)$ such that $Q_{u}(\phi)<0$ for any $\phi \in X_{K} \backslash\{0\}$. Hence, $Q_{u}(\psi) \geq 0$ for every $\psi \in C_{c}^{k}\left(\mathbb{R}^{n} \backslash \mathcal{K}\right)$, where $\mathcal{K}:=\bigcup_{j=1}^{K} \operatorname{supp}\left(\phi_{j}\right)$.

To state the following result we need to introduce some notation. Let two critical exponents play an important role, namely the classical Sobolev exponent

$$
p_{s}(n, k)= \begin{cases}+\infty & \text { if } n \leq 2 k, \\ \frac{n+2 k}{n-2 k} & \text { if } n>2 k,\end{cases}
$$

and the Joseph-Lundgren exponent

$$
p_{c}(n)= \begin{cases}+\infty & \text { if } n \leq 10 \\ \frac{(n-2)^{2}-4 n+8 \sqrt{n-1}}{(n-2)(n-10)} & \text { if } n \geq 11 .\end{cases}
$$

Note that the exponent $p_{c}(n)$ is larger than the classical critical Sobolev exponent $p_{s}(n, 1)$, $n \geq 11$

Now we can state our main nonexistence results. 
Theorem 2.1 Let $u \in C^{2 k}\left(\mathbb{R}^{n}\right)$ be a stable solution of (1.1). Assume that $f$ satisfies $H_{1}$ and $H_{2}$. If $1<\theta \leq p_{s}(n, k)$, then $u \equiv 0$.

Theorem 2.2 Let $u \in C^{2 k}\left(\mathbb{R}^{n}\right)$ be a solution of (1.1) which is stable outside a compact set. Assume that $f$ satisfies $H_{1}, H_{2}$ and $H_{3}$. If $1<\theta<p_{s}(n, k)$, then $u \equiv 0$.

The next result concerns the complete classification of entire stable solutions of the second-order equation (1.1) in the supercritical case.

Theorem 2.3 Let $u \in C^{2}\left(\mathbb{R}^{n}\right)$ be a stable solution of (1.1) with $k=1$. Assume that $f$ satisfies $H_{1}$ and $H_{2}$. If $\frac{n+2}{n-2}<\theta<p_{c}(n)$, then $u \equiv 0$.

\subsection{Berestycki and Lions Liouville-type theorem}

Now, we fix in this subsection

$$
f(u)=-m u+\lambda|u|^{\theta-1} u-\mu|u|^{p-1} u,
$$

where $\lambda, \mu$ are positive constants, $m \geq 0$ and $1<\theta, p$. We will show that $u=0$ is the unique solution of equation (1.1) under some assumptions on the parameter $m, \theta$ and $p$. Also, we observe that $f$ is neither convex nor positive function in $\mathbb{R}^{n}$. Then we have the following.

Theorem 2.4 Let $u \in C^{2 k}\left(\mathbb{R}^{n}\right)$ be a stable solution of (1.1) with $f$ satisfies (2.5).

1. If $u$ is bounded and $m>0$, then $u \equiv 0$, for any $\theta \geq p>1$.

2. If $1<p<\theta$ and $1<p \leq p_{s}(n, k)$, then $u \equiv 0$.

Remark 2.3 Clearly, if $u$ is unbounded stable solution to (1.1) with $f(u)=-m u+\lambda|u|^{\theta-1} u-$ $\mu|u|^{p-1} u$ and $m>0$, then $u \equiv 0$, for any $\theta \geq p>1$ and $n<2 k$.

Also, we will show, with very few restrictions, that there exists a necessary and sufficient condition for the nonexistence solutions which are stable outside a compact set of problem like (1.1).

Theorem 2.5 Let $u \in C^{2 k}\left(\mathbb{R}^{n}\right)$ be a solution of (1.1) which is stable outside a compact set with $f$ satisfies (2.5).

1. If $m>0$ and $1<p \leq \frac{n+2 k}{n-2 k} \leq \theta$, then $u \equiv 0$.

2. If $m=0,1<p \leq \frac{n+2 k}{n-2 k} \leq \theta$ and $(p, \theta) \neq\left(\frac{n+2 k}{n-2 k}, \frac{n+2 k}{n-2 k}\right)$, then $u \equiv 0$.

\section{Auxiliary results}

In this section we prove the following lemmas and propositions, which will have a crucial role in the proof of Theorems 2.1, 2.2, 2.3, 2.4 and 2.5. Denote $B_{R}=\left\{x \in \mathbb{R}^{n}:|x|<R\right\}$. The letter $C$ will be used throughout to denote a generic positive constant, which may vary from line to line and only depends on arguments inside the parentheses or arguments which are otherwise clear from the context.

First, define a cut-off function $\varphi_{R} \in C_{c}^{4}\left(\mathbb{R}^{n}\right)$ such that $\varphi_{R} \equiv 1$ in $B_{R}, \varphi_{R} \equiv 0$ in $\mathbb{R}^{n} \backslash\left\{B_{2 R}\right\}$, $0 \leq \varphi_{R} \leq 1$ in $\mathbb{R}^{n}$ and $\left|\nabla^{\tau} \varphi_{R}\right| \leq C R^{-\tau}$ for $\tau \leq 4$ in $A_{R}=\left\{x \in \mathbb{R}^{n}, R \leq|x| \leq 2 R\right\}$.

Lemma 3.1 For any $v \in C^{8}\left(\mathbb{R}^{n}\right), m>4$ and $\epsilon>0$ arbitrary small number, there exists a constant $C_{\epsilon, m}>0$ such that 
1. $R^{-4} \int_{B_{2 R}}|\Delta v|^{2} \varphi_{R}^{2 m-4} d x \leq \epsilon^{2} \int_{B_{2 R}}\left(\Delta^{2} v\right)^{2} \varphi_{R}^{2 m} d x+\epsilon^{2} R^{-2} \int_{B_{2 R}}|\nabla(\Delta v)|^{2} \varphi_{R}^{2 m-2} d x+$ $C_{\epsilon, m} R^{-8} \int_{B_{2 R}} v^{2} \varphi_{R}^{2 m-8} d x$

2. $R^{-2} \int_{B_{2 R}}|\nabla(\Delta v)|^{2} \varphi_{R}^{2 m-2} d x \leq \epsilon \int_{B_{2 R}}\left(\Delta^{2} v\right)^{2} \varphi_{R}^{2 m} d x+C_{\epsilon, m} R^{-8} \int_{B_{2 R}} v^{2} \varphi_{R}^{2 m-8} d x$,

3. $R^{-6} \int_{B_{2 R}}|\nabla v|^{2} \varphi_{R}^{2 m-6} d x \leq \epsilon^{3} \int_{B_{2 R}}\left(\Delta^{2} v\right)^{2} \varphi_{R}^{2 m} d x+C_{\epsilon, m} R^{-8} \int_{B_{2 R}} \nu^{2} \varphi_{R}^{2 m-8} d x$,

4. $R^{-4} \int_{B_{2 R}}\left|\nabla^{2} v\right|^{2} \varphi_{R}^{2 m-4} d x \leq \epsilon^{3} \int_{B_{2 R}}\left(\Delta^{2} v\right)^{2} \varphi_{R}^{2 m} d x+C_{\epsilon, m} R^{-8} \int_{B_{2 R}} v^{2} \varphi_{R}^{2 m-8} d x$,

5. $R^{-2} \int_{B_{2 R}}\left|\nabla^{3} v\right|^{2} \varphi_{R}^{2 m-2} d x \leq \epsilon^{3} \int_{B_{2 R}}\left(\Delta^{2} v\right)^{2} \varphi_{R}^{2 m} d x+C_{\epsilon, m} R^{-8} \int_{B_{2 R}} v^{2} \varphi_{R}^{2 m-8} d x$.

Proof Fix $m>4$. Let $v \in C^{8}\left(\mathbb{R}^{n}\right)$ and $\varphi_{R} \in C_{c}^{4}\left(\mathbb{R}^{n}\right)$ defined as above.

Proof of 1 . Integrating by parts, we get

$$
\begin{aligned}
R^{-4} & \int_{B_{2 R}}(\Delta \nu)^{2} \varphi_{R}^{2 m-4} d x \\
& =R^{-4} \int_{B_{2 R}} v\left(\Delta^{2} v \varphi_{R}^{2 m-4}+\Delta v \Delta\left(\varphi_{R}^{2 m-4}\right)+2 \nabla(\Delta v) \nabla\left(\varphi_{R}^{2 m-4}\right)\right) d x .
\end{aligned}
$$

An application of Young's inequality yields

$$
\begin{aligned}
R^{-4} & \int_{B_{2 R}} v\left(\Delta^{2} v \varphi_{R}^{2 m-4}+\Delta v \Delta\left(\varphi_{R}^{2 m-4}\right)+2 \nabla(\Delta v) \nabla\left(\varphi_{R}^{2 m-4}\right)\right) d x \\
\leq & \epsilon^{2} \int_{B_{2 R}}\left(\Delta^{2} v\right)^{2} \varphi_{R}^{2 m} d x+\epsilon^{2} R^{-2} \int_{B_{2 R}}|\nabla(\Delta v)|^{2} \varphi_{R}^{2 m-2} d x \\
& +\frac{R^{-4}}{2} \int_{B_{2 R}}(\Delta v)^{2} \varphi_{R}^{2 m-4} d x+C_{\epsilon, m} R^{-8} \int_{B_{2 R}} v^{2} \varphi_{R}^{2 m-8} d x
\end{aligned}
$$

Inserting the latter inequality into (3.1), we obtain

$$
\begin{aligned}
R^{-4} \int_{B_{2 R}}(\Delta v)^{2} \varphi_{R}^{2 m-4} d x \leq & \epsilon^{2} \int_{B_{2 R}}\left(\Delta^{2} v\right)^{2} \varphi_{R}^{2 m} d x+\epsilon^{2} R^{-2} \int_{B_{2 R}}|\nabla(\Delta v)|^{2} \varphi_{R}^{2 m-2} d x \\
& +C_{\epsilon, m} R^{-8} \int_{B_{2 R}} v^{2} \varphi_{R}^{2 m-8} d x .
\end{aligned}
$$

Proof of 2. Integrating by parts and using again Young's inequality, we obtain

$$
\begin{aligned}
& R^{-2} \int_{B_{2 R}}|\nabla(\Delta v)|^{2} \varphi_{R}^{2 m-2} d x \\
& \quad=-R^{-2} \int_{B_{2 R}} \Delta v \Delta^{2} v \varphi_{R}^{2 m-2} d x-R^{-2} \int_{B_{2 R}} \Delta v \nabla(\Delta v) \nabla\left(\varphi_{R}^{2 m-2}\right) d x \\
& \quad \leq \epsilon \int_{B_{2 R}}\left(\Delta^{2} v\right)^{2} \varphi_{R}^{2 m} d x+\frac{2 R^{-4}}{\epsilon} \int_{B_{2 R}}(\Delta v)^{2} \varphi_{R}^{2 m-4} d x+C \epsilon R^{-2} \int_{B_{2 R}}|\nabla(\Delta v)|^{2} \varphi_{R}^{2 m-2} d x .
\end{aligned}
$$

Inserting (3.2) into the latter, we derive

$$
R^{-2} \int_{B_{2 R}}|\nabla(\Delta v)|^{2} \varphi_{R}^{2 m-2} d x \leq \epsilon \int_{B_{2 R}}\left(\Delta^{2} v\right)^{2} \varphi_{R}^{2 m} d x+C_{\epsilon, m} R^{-8} \int_{B_{2 R}} v^{2} \varphi_{R}^{2 m-8} d x .
$$

Proof of 3. Integrating by parts, we obtain

$$
\begin{aligned}
\int_{B_{2 R}}|\nabla v|^{2} \varphi_{R}^{2 m-6} d x & =\frac{R^{-6}}{2} \int_{B_{2 R}} \Delta\left(v^{2}\right) \varphi_{R}^{2 m-6} d x-R^{-6} \int_{B_{2 R}} v \Delta v \varphi_{R}^{2 m-6} d x \\
& \leq \epsilon R^{-4} \int_{B_{2 R}}(\Delta v)^{2} \varphi_{R}^{2 m-4} d x+C_{\epsilon, m} R^{-8} \int_{B_{2 R}} v^{2} \varphi_{R}^{2 m-8} d x
\end{aligned}
$$


From (3.2) and (3.3), we deduce

$$
R^{-6} \int_{B_{2 R}}|\nabla v|^{2} \varphi_{R}^{2 m-6} d x \leq \epsilon^{3} \int_{B_{2 R}}\left(\Delta^{2} v\right)^{2} \varphi_{R}^{2 m} d x+C_{\epsilon, m} R^{-8} \int_{B_{2 R}} v^{2} \varphi_{R}^{2 m-8} d x .
$$

Proof of 4 . Integrating by parts, we obtain

$$
\begin{aligned}
& R^{-4} \int_{B_{2 R}}\left|\nabla^{2} v\right|^{2} \varphi_{R}^{2 m-4} d x \\
& \quad=-R^{-4} \int_{B_{2 R}} \nabla v \nabla(\Delta v) \varphi_{R}^{2 m-4} d x+\frac{R^{-4}}{2} \int_{B_{2 R}}|\nabla v|^{2} \Delta\left(\varphi_{R}^{2 m-4}\right) d x .
\end{aligned}
$$

Using Young's inequality and from (3.3) and (3.4), we obtain

$$
\begin{aligned}
R^{-4} \int_{B_{2 R}}\left|\nabla^{2} v\right|^{2} \varphi_{R}^{2 m-4} d x & \leq R^{-2} \int_{B_{2 R}}|\nabla(\Delta v)|^{2} \varphi_{R}^{2 m-2} d x+C R^{-6} \int_{B_{2 R}}|\nabla v|^{2} \varphi_{R}^{2 m-6} d x \\
& \leq \epsilon^{3} \int_{B_{2 R}}\left(\Delta^{2} v\right)^{2} \varphi_{R}^{2 m}+C_{\epsilon, m} R^{-8} \int_{B_{2 R}} v^{2} \varphi_{R}^{2 m-8} .
\end{aligned}
$$

Proof of 5. Integrating by parts, we get

$$
\begin{aligned}
R^{-2} & \int_{B_{2 R}}\left|\nabla^{3} v\right|^{2} \varphi_{R}^{2 m-2} d x \\
\quad= & R^{-2} \int_{B_{2 R}}\left(|\nabla(\Delta v)|^{2} \varphi_{R}^{2 m-2}+v_{i j} \Delta v_{i}\left(\varphi_{R}^{2 m-2}\right)_{j}+\frac{1}{2}\left|\nabla^{2} v\right|^{2} \Delta\left(\varphi_{R}^{2 m-2}\right)\right) d x,
\end{aligned}
$$

where $f_{i}=\frac{\partial f}{\partial x_{i}}, f_{i j}=\frac{\partial^{2} f}{\partial x_{j} \partial x_{i}}$ and $f_{i j k}=\frac{\partial^{3} f}{\partial x_{k} \partial x_{j} \partial x_{i}}$. (Here and in the sequel, we use the Einstein summation convention: an index occurring twice in a product is to be summed from $1 \mathrm{up}$ to the space dimension.)

Using Young's inequality of the above, we deduce

$$
\begin{aligned}
& R^{-2} \int_{B_{2 R}}\left|\nabla^{3} v\right|^{2} \varphi_{R}^{2 m-2} d x \\
& \quad \leq C R^{-2} \int_{B_{2 R}}|\nabla(\Delta v)|^{2} \varphi_{R}^{2 m-2} d x+C R^{-4} \int_{B_{2 R}}\left|\nabla^{2} v\right|^{2} \varphi_{R}^{2 m-4} d x,
\end{aligned}
$$

which gives the desired conclusion.

Lemma 3.2 For any $m>4$ and $\epsilon>0$ arbitrary small number, there exists a constant $C_{\epsilon, m}>0$ such that

$$
\left(\Delta^{2}\left(u \varphi_{R}^{m}\right)\right)^{2} \leq(1+\epsilon)\left(\varphi_{R}^{m} \Delta^{2} u\right)^{2}+C_{\epsilon, m} \mathbf{B}\left(u, \varphi_{R}, m\right)
$$

where $\mathbf{B}\left(u, \varphi_{R}, m\right)=\left(R^{-4}|\Delta u|^{2} \varphi_{R}^{2 m-4}+R^{-2}|\nabla(\Delta u)|^{2} \varphi_{R}^{2 m-2}+R^{-6}|\nabla u|^{2} \varphi_{R}^{2 m-6}+R^{-8} u^{2} \varphi_{R}^{2 m-8}+\right.$ $\left.R^{-4}\left|\nabla^{2} u\right|^{2} \varphi_{R}^{2 m-4}\right)$.

Proof Let $\varphi_{R} \in C_{c}^{4}\left(\mathbb{R}^{n}\right)$ be defined as above and $m>4$. Direct calculation yields

$$
\Delta^{2}\left(u \varphi_{R}^{m}\right)=\varphi_{R}^{m} \Delta^{2} u+\mathbf{A}\left(u, \varphi_{R}^{m}\right)
$$

where $\mathbf{A}\left(u, \varphi_{R}^{m}\right)=2 \Delta u \Delta \varphi_{R}^{m}+4 \nabla u \nabla\left(\Delta \varphi_{R}^{m}\right)+u \Delta^{2} \varphi_{R}^{m}+4 \nabla(\Delta u) \nabla\left(\varphi_{R}^{m}\right)+4 u_{i j}\left(\varphi_{R}^{m}\right)_{i j}$. 
Thus,

$$
\left(\Delta^{2}\left(u \varphi_{R}^{m}\right)\right)^{2}=\left(\varphi_{R}^{m} \Delta^{2} u\right)^{2}+\mathbf{A}^{2}\left(u, \varphi_{R}^{m}\right)+2 \mathbf{A}\left(u, \varphi_{R}^{m}\right) \varphi_{R}^{m} \Delta^{2} u
$$

Now by the Young inequality, for any $\epsilon>0$, there exists $C_{\epsilon}$ a constant such that

$$
\left(\Delta^{2}\left(u \varphi_{R}^{m}\right)\right)^{2} \leq(1+\epsilon)\left(\varphi_{R}^{m} \Delta^{2} u\right)^{2}+C_{\epsilon} \mathbf{A}^{2}\left(u, \varphi_{R}^{m}\right)
$$

For the second term on the right hand side of inequality (3.9), one obtains

$$
\begin{aligned}
\mathbf{A}^{2}\left(u, \varphi_{R}^{m}\right) \leq & C_{\epsilon}\left(|\Delta u|^{2}\left|\Delta \varphi_{R}^{m}\right|^{2}+|\nabla u|^{2}\left|\nabla\left(\Delta \varphi_{R}^{m}\right)\right|^{2}+|u|^{2}\left|\Delta^{2} \varphi_{R}^{m}\right|^{2}\right. \\
& \left.+|\nabla(\Delta u)|^{2}\left|\nabla\left(\varphi_{R}^{m}\right)\right|^{2}+\left|u_{i j}\right|^{2}\left|\left(\varphi_{R}^{m}\right)_{i j}\right|^{2}\right) \\
\leq & C_{\epsilon, m}\left(R^{-4}|\Delta u|^{2} \varphi_{R}^{2 m-4}+R^{-2}|\nabla(\Delta u)|^{2} \varphi_{R}^{2 m-2}+R^{-6}|\nabla u|^{2} \varphi_{R}^{2 m-6}\right. \\
& \left.+R^{-8} u^{2} \varphi_{R}^{2 m-8}+R^{-4}\left|\nabla^{2} u\right|^{2} \varphi_{R}^{2 m-4}\right),
\end{aligned}
$$

which gives the desired inequality (3.7).

Using the previous lemmas, we obtain the following results.

Proposition 3.1 Let $u \in C^{2 k}\left(\mathbb{R}^{n}\right)$ be a stable solution of (1.1). Assume that $f$ satisfies $H_{1}$ and $\mathrm{H}_{2}$. Then there exists a constant $\mathrm{C}>0$ such that, for any $\mathrm{R}>0$, we have

$$
\int_{B_{R}}\left(|u|^{\theta+1}+\left|D^{k} u\right|^{2}\right) d x \leq C R^{n-2 k_{\theta-1}^{\theta+1}} \text { and } \quad \int_{B_{R}} f(u) u d x \leq C R^{n-2 k \frac{\theta+1}{\theta-1}}
$$

When attempting to prove the nonexistence of the nontrivial solution which is stable outside a compact set of (1.1) in the subcritical case, we need first to establish the following proposition.

Proposition 3.2 Let $u \in C^{2 k}\left(\mathbb{R}^{n}\right)$ be a solution of (1.1) which is stable outside a compact set. Assume that $f$ satisfies $H_{1}$ and $H_{2}$. Then there exists a constant $C>0$ such that, for any $R>0$, we have

$$
\int_{B_{R}}\left(|u|^{\theta+1}+\left|D^{k} u\right|^{2}\right) d x \leq C\left(1+R^{n-2 k^{\frac{\theta+1}{\theta-1}}}\right) \quad \text { and } \quad \int_{B_{R}} f(u) u d x \leq C\left(1+R^{n-2 k \frac{\theta+1}{\theta-1}}\right) .
$$

Proof of Proposition 3.1 The proof of the case $k=1,2,3$, bears resemblance to an argument found in $[5,6,8]$. For more details, please see the proof of proposition 4 in [6] for the case $k=1$, the proof of Lemma 4.2 in [5] for the case $k=2$ and the proof of Proposition 1.2 in [8] for the case $k=3$. For this reason, we omit the details.

Proof of the case $k=4$. Let $\varphi_{R} \in C_{c}^{4}\left(\mathbb{R}^{n}\right)$ defined as above, let $u$ be a solution of equation (1.1). The function $u \varphi_{R}^{m}$ belongs to $C_{c}^{4}\left(\mathbb{R}^{n}\right)$, and thus it can be used as a test function in the quadratic form $Q_{u}$. Hence, the stability assumption on $u$ gives

$$
\int_{B_{2 R}} f^{\prime}(u) u^{2} \varphi_{R}^{2 m} d x \leq \int_{B_{2 R}}\left|\Delta^{2}\left(u \varphi_{R}^{m}\right)\right|^{2} d x .
$$


Applying Lemma 3.2, we obtain

$$
\int_{B_{2 R}} f^{\prime}(u) u^{2} \varphi_{R}^{2 m} d x \leq(1+\epsilon) \int_{B_{2 R}}\left(\varphi_{R}^{m} \Delta^{2} u\right)^{2} d x+C_{\epsilon} \int_{B_{2 R}} \mathbf{B}\left(u, \varphi_{R}, m\right) d x .
$$

In view of Lemma 3.1, we get

$$
\int_{B_{2 R}} f^{\prime}(u) u^{2} \varphi_{R}^{2 m} d x \leq(1+\epsilon) \int_{B_{2 R}}\left(\varphi_{R}^{m} \Delta^{2} u\right)^{2}+C_{\epsilon} R^{-8} \int_{B_{2 R}} u^{2} \varphi_{R}^{2 m-8} d x .
$$

Multiplying equation (1.1) by $u \varphi_{R}^{2 m}$ and integrating by parts, we get

$$
\int_{B_{2 R}} \Delta^{2} u \Delta^{2}\left(u \varphi_{R}^{2 m}\right) d x=\int_{B_{2 R}} f(u) u \varphi_{R}^{2 m} d x
$$

From (3.8), we derive

$$
\begin{aligned}
& \int_{B_{2 R}} \Delta^{2} u \Delta^{2}\left(u \varphi_{R}^{2 m}\right) d x \\
& =\int_{B_{2 R}} \Delta^{2} u\left\{\left(\Delta^{2} u\right) \varphi_{R}^{2 m}+2 \Delta u \Delta\left(\varphi_{R}^{2 m}\right)+4 u_{i j}\left(\varphi_{R}^{2 m}\right)_{i j}\right. \\
& \left.\quad+4 \nabla(\Delta u) \nabla\left(\varphi_{R}^{2 m}\right)+4 \nabla u \nabla\left(\Delta\left(\varphi_{R}^{2 m}\right)\right)+u \Delta^{2}\left(\varphi_{R}^{2 m}\right)\right\} d x,
\end{aligned}
$$

therefore

$$
\int_{B_{2 R}}\left(\left(\Delta^{2} u\right)^{2} \varphi_{R}^{2 m}-f(u) u \varphi_{R}^{2 m}\right) d x=-\int_{B_{2 R}} \Delta^{2} u \mathbf{A}\left(u, \varphi_{R}^{2 m}\right) d x .
$$

Then, using Young's inequality, we derive

$$
\int_{B_{2 R}}\left(\left(\Delta^{2} u\right)^{2} \varphi_{R}^{2 m}-f(u) u \varphi_{R}^{2 m}\right) d x \leq \epsilon \int_{B_{2 R}}\left(\Delta^{2} u\right)^{2} \varphi_{R}^{2 m} d x+C_{\epsilon, m} \int_{B_{2 R}} \mathbf{B}\left(u, \varphi_{R}, m\right) d x .
$$

Applying again Lemma 3.1, we have

$$
\begin{aligned}
& \int_{B_{2 R}}\left(\Delta^{2} u\right)^{2} \varphi_{R}^{2 m} d x-\int_{B_{2 R}} f(u) u \varphi_{R}^{2 m} d x \\
& \quad \leq \epsilon \int_{B_{2 R}}\left(\Delta^{2} u\right)^{2} \varphi_{R}^{2 m} d x+C_{\epsilon, m} R^{-8} \int_{B_{2 R}} u^{2} \varphi_{R}^{2 m-8} d x
\end{aligned}
$$

Multiplying (3.13) by $\theta$ and combining it with (3.11), we derive

$$
\begin{aligned}
& \int_{B_{2 R}}\left[f^{\prime}(u) u^{2}-\theta f(u) u\right] \varphi_{R}^{2 m} d x+[\theta(1-\epsilon)-(1+\epsilon)] \int_{B_{2 R}}\left(\Delta^{2} u\right)^{2} \varphi_{R}^{2 m} d x \\
& \quad \leq C R^{-8} \int_{B_{2 R}} u^{2} \varphi_{R}^{2 m-8} d x .
\end{aligned}
$$

From $\left(H_{1}\right)$ and for $\epsilon$ sufficiently small such that $\epsilon<\frac{\theta-1}{\theta+1}$, we deduce

$$
\int_{B_{2 R}}\left(\Delta^{2} u\right)^{2} \varphi_{R}^{2 m} d x \leq C R^{-8} \int_{B_{2 R}} u^{2} \varphi_{R}^{2 m-8} d x
$$


By Young's inequality, we have

$$
\int_{B_{2 R}}\left(\Delta^{2} u\right)^{2} \varphi_{R}^{2 m} d x \leq \frac{2}{\theta+1} \int_{B_{2 R}}|u|^{\theta+1} \varphi_{R}^{(\theta+1)(m-4)} d x+C R^{n-8 \frac{\theta+1}{\theta-1}}
$$

As above, we find from (3.13) that

$$
\int_{B_{2 R}} f(u) u \varphi_{R}^{2 m} d x \leq(1+\epsilon) \int_{B_{2 R}}\left(\Delta^{2} u\right)^{2} \varphi_{R}^{2 m} d x+C_{\epsilon, m} R^{-8} \int_{B_{2 R}} u^{2} \varphi_{R}^{2 m-8} d x .
$$

Using (3.14) in the latter, we obtain

$$
\begin{aligned}
\int_{B_{2 R}} f(u) u \varphi_{R}^{2 m} d x & \leq C_{\epsilon} R^{-8} \int_{B_{2 R}} u^{2} \varphi_{R}^{2 m-8} d x \\
& \leq \frac{2}{\theta+1} \int_{B_{2 R}}|u|^{\theta+1} \varphi_{R}^{(\theta+1)(m-4)} d x+C R^{n-8 \frac{\theta+1}{\theta-1}}
\end{aligned}
$$

From $\left(H_{1}^{\prime}\right)$ and $\left(H_{2}\right)$, we get

$$
\begin{aligned}
C_{0} \int_{B_{2 R}}|u|^{\theta+1} \varphi_{R}^{2 m} d x & \leq \int_{B_{2 R}} f(u) u \varphi_{R}^{2 m} d x \\
& \leq \frac{2}{\theta+1} \int_{B_{2 R}}|u|^{\theta+1} \varphi_{R}^{(\theta+1)(m-4)} d x+C R^{n-8 \frac{\theta+1}{\theta-1}},
\end{aligned}
$$

if $(\theta+1)(m-4)=2 m$, then

$$
\int_{B_{2 R}}|u|^{\theta+1} \varphi_{R}^{2 m} d x \leq C R^{n-8 \frac{\theta+1}{\theta-1}}
$$

From (3.15), (3.16) and (3.17), we deduce that

$$
\int_{B_{2 R}}\left(\Delta^{2} u\right)^{2} \varphi_{R}^{2 m} d x \leq C R^{n-8 \frac{\theta+1}{\theta-1}}, \quad \text { and } \quad \int_{B_{2 R}} f(u) u \varphi_{R}^{2 m} d x \leq C R^{n-8} \frac{\theta+1}{\theta-1}
$$

Since $\varphi_{R} \equiv 1$ in $B_{R}$, we have

$$
\int_{B_{R}}\left(|u|^{\theta+1}+\left(\Delta^{2} u\right)^{2}\right) d x \leq C R^{n-8 \frac{\theta+1}{\theta-1}}, \quad \text { and } \quad \int_{B_{R}} f(u) u d x \leq C R^{n-8 \frac{\theta+1}{\theta-1}}
$$

Proof of Proposition 3.2 The proof of the case $k=1,2,3$, bears resemblance to an argument found in $[5,6,8]$. Now, we prove the case $k=4$. The proof is the same as the proof of Proposition 3.1. We need only to replace $\varphi_{R}$ by $\varphi_{a, R}$, where $\varphi_{a, R} \in C_{c}^{4}\left(\mathbb{R}^{n}\right)$ satisfies $0 \leq$ $\varphi_{a, R} \leq 1$ everywhere on $\mathbb{R}^{n}$ such that $\varphi_{a, R}(x)=0$ for $|x|<a$ or $|x|>2 R, \varphi_{a, R}(x)=1$ for $2 a<$ $|x|<R$ and $\left|\nabla^{\tau} \varphi_{a_{0}, R}\right| \leq C R^{-\tau}, \tau \leq 4$, for $R<|x|<2 R$. By the stability assumption on $u$, there exists $a_{0}>0$ such that $Q_{u}\left(u \varphi_{a_{0}, R}^{m}\right) \geq 0$ for any $R>2 a_{0}$. Hence, by the choice of the test function $\varphi_{a, R}$, the constant $C_{a_{0}}$ depending on $a_{0}, \epsilon, m$ and $u$ appears and the rest of the proof is unchanged. Thus Proposition 3.2 follows.

As in [20], we shall employ a cut-off function with compact support to derive a variant of the Pohozaev identity. This device allows us to avoid the spherical integrals raised in 
[21], which are very difficult to control, especially for the polyharmonic situations. For $k=1,2,3$, the Pohozaev identity is similar to $[7,8,20,22]$.

Proposition 3.3 Let $u \in C^{8}\left(\mathbb{R}^{n}\right)$ be a solution of (1.1) and $\psi \in C_{c}^{4}\left(B_{R}\right)$, then

$$
\frac{n-8}{2} \int_{B_{R}}\left(\Delta^{2} u\right)^{2} \psi d x-n \int_{B_{R}} F(u) \psi d x=\int_{B_{R}} B_{4}(u, \psi) d x,
$$

where

$$
\begin{aligned}
B_{4}(u, \psi)= & F(u)\langle x, \nabla \psi\rangle-\frac{1}{2}\left(\Delta^{2} u\right)^{2}\langle x, \nabla \psi\rangle+2 \Delta^{2} u \nabla(\langle x, \nabla(\Delta u)|) \nabla \psi \\
& +\Delta^{2} u\{\langle x, \nabla(\Delta u)\rangle \Delta \psi+2 \Delta u \Delta \psi\}+\Delta^{2} u\left\{4 \nabla(\Delta u) \nabla \psi+\Delta^{2} \psi\langle x, \nabla u\rangle\right\} \\
& +\Delta^{2} u\{\Delta \psi \Delta(\langle x, \nabla u\rangle)+2 \nabla(\Delta \psi) \nabla(\langle x, \nabla u\rangle)+2 \Delta[\nabla(\langle x, \nabla u\rangle) \nabla \psi]\} .
\end{aligned}
$$

Thanks to Propositions 3.2 and 3.3, we derive the following.

Proposition 3.4 Let $u \in C^{2 k}\left(\mathbb{R}^{n}\right)$ be a solution of (1.1) which is stable outside a compact set. Assume that $f$ satisfies $H_{1}$ and $H_{2}$. If $1<\theta<p_{s}(n, k)$, then

$$
\int_{\mathbb{R}^{n}}\left|D^{k} u\right|^{2} d x=\frac{2 n}{n-2 k} \int_{\mathbb{R}^{n}} F(u) d x
$$

and

$$
\int_{\mathbb{R}^{n}}\left|D^{k} u\right|^{2} d x=\int_{\mathbb{R}^{n}} f(u) u d x<\infty .
$$

Proof of Proposition 3.3 Let $u \in C^{8}\left(\mathbb{R}^{n}\right)$ be a solution of (1.1) and $\psi \in C_{c}^{4}\left(B_{R}\right)$, we have

$$
\Delta(\langle x, \nabla u\rangle \psi)=\langle x, \nabla(\Delta u)\rangle \psi+2 \Delta u \psi+\langle x, \nabla u\rangle \Delta \psi+2 \nabla(\langle x, \nabla u\rangle) \nabla \psi .
$$

Multiplying equation (1.1) by $\langle x, \nabla u\rangle \psi$ and integrating by parts in $B_{R}$, we obtain

$$
\int_{B_{R}} f(u)\langle x, \nabla u\rangle \psi d x=\int_{B_{R}} \Delta^{3} u \Delta(\langle x, \nabla u\rangle \psi) d x .
$$

For the right hand side of (3.21), we integrate by parts to get

$$
\begin{aligned}
\int_{B_{R}} & \Delta^{3} u \Delta(\langle x, \nabla u\rangle \psi) d x \\
= & \int_{B_{R}} \Delta^{3} u(\langle x, \nabla(\Delta u)\rangle \psi+2 \Delta u \psi+\langle x, \nabla u\rangle \Delta \psi+2 \nabla(\langle x, \nabla u\rangle) \nabla \psi) d x \\
= & \int_{B_{R}} \Delta^{2} u \Delta[\langle x, \nabla(\Delta u)\rangle] \psi d x+2 \int_{B_{R}} \Delta^{2} u \nabla[\langle x, \nabla(\Delta u)\rangle] \nabla \psi d x+2 \int_{B_{R}}\left(\Delta^{2} u\right)^{2} \psi d x \\
& +\int_{B_{R}} \Delta^{2} u\left\{\langle x, \nabla(\Delta u)\rangle \Delta \psi+2 \Delta u \Delta \psi+4 \nabla(\Delta u) \nabla \psi+\langle x, \nabla u\rangle \Delta^{2} \psi\right\} d x \\
& +\int_{B_{R}} \Delta^{2} u\{\Delta[\langle x, \nabla u\rangle] \Delta \psi+2 \nabla[\langle x, \nabla u\rangle] \nabla(\Delta \psi)\} d x \\
& +2 \int_{B_{R}} \Delta^{2} u \Delta[\nabla(\langle x, \nabla u\rangle) \nabla \psi] d x
\end{aligned}
$$


For the first term on the right hand side of (3.22), we integrate by parts to find

$$
\int_{B_{R}} \Delta^{2} u \Delta[\langle x, \nabla(\Delta u)\rangle] \psi d x=\frac{4-n}{2} \int_{B_{R}}\left(\Delta^{2} u\right)^{2} \psi d x-\frac{1}{2} \int_{B_{R}}\left(\Delta^{2} u\right)^{2}\langle x, \nabla \psi\rangle d x .
$$

For the term on the left hand side of (3.22), by integrating by parts, we derive

$$
\begin{aligned}
\int_{B_{R}} f(u)\langle x, \nabla u\rangle \psi d x & =\int_{B_{R}}\langle x, \nabla[F(u)]\rangle \psi d x \\
& =-n \int_{B_{R}} F(u) \psi d x-\int_{B_{R}} F(u)\langle x, \nabla \psi\rangle d x .
\end{aligned}
$$

Therefore, the claim follows from (3.21)-(3.24).

Here, we are concerned with the proof of Proposition 3.4.

Proof of Proposition 3.4 To simplify the proof, we will concentrate on the case $k=4$ which is the most delicate case; even we believe that the results should hold true for $k=1,2,3$, for more details, see for example $[5,6,8,23]$. Let $R_{0}>0$. Assume that $u$ is stable outside $B_{R_{0}}$. Let $0<\alpha<\beta$. We begin by defining some smooth compactly supported functions which will be used several times in the sequel. More precisely, we choose $\phi_{R} \in C_{c}^{4}\left(\mathbb{R}^{n}\right)$ satisfies $0 \leq \phi_{R} \leq 1$ everywhere on $\mathbb{R}^{n}$ such that

$$
\phi_{R}(x)= \begin{cases}1 & \text { for } \alpha R<|x|<\beta R, \\ 0 & \text { for }|x|<\frac{\alpha}{2} R \text { or }|x|>2 \beta R, \\ \left|\nabla^{k} \phi_{R}\right| \leq C R^{-k} & \text { on }\left\{\frac{\alpha}{2} R<|x|<2 \beta R\right\}, k=1,2,3,4 .\end{cases}
$$

For $R$ large enough such that $\frac{\alpha}{2} R>R_{0}$, then $B_{R_{0}} \cap\left\{\frac{\alpha}{2} R \leq|x| \leq 2 \beta R\right\}=\varnothing$. Then $u$ is stable in $A_{\frac{\alpha}{2} R}^{2 \beta R}:=\left\{\frac{\alpha}{2} R<|x|<2 \beta R\right\}$. By Proposition 3.1, there exists a constant $C>0$ such that

$$
\int_{A_{\alpha R}^{\beta R}}\left(|u|^{\theta+1}+\left(\Delta^{2} u\right)^{2}\right) d x \leq C R^{n-8 \frac{\theta+1}{\theta-1}} \quad \text { and } \quad \int_{A_{\alpha R}^{\beta R}} f(u) u d x \leq C R^{n-8 \frac{\theta+1}{\theta-1}} .
$$

Let $\psi_{R} \in C_{c}^{4}\left(\mathbb{R}^{n}\right)$ satisfies $0 \leq \psi_{R} \leq 1$ on $\mathbb{R}^{n}$ defined by

$$
\psi_{R}(x)= \begin{cases}1 & \text { for }|x|<\alpha R \\ 0 & \text { for }|x|>\beta R, \\ \left|\nabla^{k} \psi_{R}\right| \leq C R^{-k} & \text { on }\{\alpha R<|x|<\beta R\}, k=1,2,3,4 .\end{cases}
$$

In view of Lemma 3.1 and Proposition 3.1, we have

$$
\begin{aligned}
& \int_{B_{\beta R}}\left(|u|^{\theta+1} \psi_{R}^{2 m}+\left(\Delta^{2} u\right)^{2} \psi_{R}^{2 m}\right) d x \leq C R^{n-8 \frac{\theta+1}{\theta-1}} \\
& \int_{B_{\beta R}}\left(\mathbf{B}\left(u, \psi_{R}, m\right)+R^{-2}\left|\nabla^{3} u\right|^{2} \psi_{R}^{2 m-2}\right) d x \leq C R^{n-8 \frac{\theta+1}{\theta-1}}
\end{aligned}
$$

Now, we estimate all terms on the right hand side of (3.18). Take $\psi=\psi_{R}^{2 m}$ in (3.18), $m>4$. 
The second term on the right hand side of (3.18) can be estimated as

$$
\begin{aligned}
\left|-\frac{1}{2} \int_{B_{\beta R}}\left(\Delta^{2} u\right)^{2}\left\langle x, \nabla \psi_{R}^{2 m}\right\rangle d x\right| & =\left|-\frac{1}{2} \int_{A_{\alpha R}^{\beta R}}\left(\Delta^{2} u\right)^{2}\left\langle x, \nabla \psi_{R}^{2 m}\right\rangle d x\right| \\
& \leq C_{m} \int_{A_{\alpha R}^{\beta R}}\left(\Delta^{2} u\right)^{2} \psi_{R}^{2 m-1} d x \leq C R^{n-8 \frac{\theta+1}{\theta-1}}
\end{aligned}
$$

Next

$$
\begin{aligned}
& \mid \int_{B_{\beta R}}\left[\Delta^{2} u\left(|x, \nabla(\Delta u)\rangle \Delta \psi_{R}^{2 m}+2 \Delta u \Delta \psi_{R}^{2 m}+4 \nabla(\Delta u) \nabla \psi_{R}^{2 m}+\Delta^{2} \psi_{R}^{2 m}\langle x, \nabla u\rangle\right)\right] d x \mid \\
& \quad=\mid \int_{A_{\alpha R}^{\beta R}}\left[\Delta^{2} u\left(|x, \nabla(\Delta u)\rangle \Delta \psi_{R}^{2 m}+2 \Delta u \Delta \psi_{R}^{2 m}+4 \nabla(\Delta u) \nabla \psi_{R}^{2 m}+\Delta^{2} \psi_{R}^{2 m}\langle x, \nabla u\rangle\right)\right] d x \mid \\
& \quad \leq C_{m} \int_{A_{\alpha R}^{\beta R}}\left|\Delta^{2} u\right|\left(R^{-1}|\nabla(\Delta u)| \psi_{R}^{2 m-2}+R^{-2}|\Delta u| \psi_{R}^{2 m-2}+R^{-3}|\nabla u| \psi_{R}^{2 m-4}\right) d x \\
& \quad \leq C_{m} \int_{A_{\alpha R}^{\beta R}}\left|\Delta^{2} u\right|\left(R^{-1}|\nabla(\Delta u)| \psi_{R}^{m-1}+R^{-2}|\Delta u| \psi_{R}^{m-2}+R^{-3}|\nabla u| \psi_{R}^{m-3}\right) d x,
\end{aligned}
$$

the last line comes from the fact that $0 \leq \psi_{R} \leq 1$, hence $\psi_{R}^{s} \leq \psi_{R}^{t}$, for any $t \leq s$.

By applying the Hölder inequality and the Young inequality to (3.29), we have

$$
\begin{aligned}
& \mid \int_{B_{\beta R}}\left[\Delta^{2} u\left(|x, \nabla(\Delta u)\rangle \Delta \psi_{R}^{2 m}+2 \Delta u \Delta \psi_{R}^{2 m}+4 \nabla(\Delta u) \nabla \psi_{R}^{2 m}+\Delta^{2} \psi_{R}^{2 m}\langle x, \nabla u\rangle\right)\right] d x \mid \\
& \leq \int_{A_{\alpha R}^{\beta R}}\left|\Delta^{2} u\right|\left(R^{-1}|\nabla(\Delta u)| \psi_{R}^{m-1}+R^{-2}|\Delta u| \psi_{R}^{m-2}+R^{-3}|\nabla u| \psi_{R}^{m-3}\right) d x \\
& \leq\left(\int_{A_{\alpha R}^{\beta R}}\left(\Delta^{2} u\right)^{2} d x\right)^{\frac{1}{2}}\left(\int _ { A _ { \alpha R } ^ { \beta R } } \left(R^{-1}|\nabla(\Delta u)| \psi_{R}^{m-1}+R^{-2}|\Delta u| \psi_{R}^{m-2}\right.\right. \\
& \left.\left.\quad+R^{-3}|\nabla u| \psi_{R}^{m-3}\right)^{2} d x\right)^{\frac{1}{2}} \\
& \quad C\left(\int_{A_{\alpha R}^{\beta R}}\left(\Delta^{2} u\right)^{2} d x\right)^{\frac{1}{2}}\left(\int _ { A _ { \alpha R } ^ { \beta R } } \left(R^{-2}|\nabla(\Delta u)|^{2} \psi_{R}^{2 m-2}+R^{-4}|\Delta u|^{2} \psi_{R}^{2 m-4}\right.\right. \\
& \left.\left.\quad+R^{-6}|\nabla u|^{2} \psi_{R}^{2 m-6}\right) d x\right)^{\frac{1}{2}} .
\end{aligned}
$$

Similarly, we also obtain

$$
\begin{aligned}
& \left|\int_{B_{\beta R}} \Delta^{2} u \nabla(|x, \nabla(\Delta u)|) \nabla \psi_{R}^{2 m} d x\right| \\
& \quad=\left|\int_{B_{\beta R}} \Delta^{2} u\left(\nabla(\Delta u) \nabla \psi_{R}^{2 m}+x_{i}(\Delta u)_{i j}\left(\psi_{R}^{2 m}\right)_{j}\right) d x\right| \\
& \quad \leq C_{m} \int_{A_{\alpha R}^{\beta R}}\left|\Delta^{2} u\right|\left(R^{-1}|\nabla(\Delta u)| \psi_{R}^{2 m-1}+\left|(\Delta u)_{i j}\right| \psi_{R}^{2 m-1}\right) d x \\
& \quad \leq C\left(\int_{A_{\alpha R}^{\beta R}}\left(\Delta^{2} u\right)^{2} d x\right)^{\frac{1}{2}}\left(\int_{A_{\alpha R}^{\beta R}}\left(R^{-1}|\nabla(\Delta u)| \psi_{R}^{2 m-1}+\left|(\Delta u)_{i j}\right| \psi_{R}^{2 m-1}\right)^{2} d x\right)^{\frac{1}{2}} \\
& \quad \leq C\left(\int_{A_{\alpha R}^{\beta R}}\left(\Delta^{2} u\right)^{2} d x\right)^{\frac{1}{2}}\left(\int_{A_{\alpha R}^{\beta R}}\left(R^{-2}|\nabla(\Delta u)|^{2} \psi_{R}^{4 m-2}+\left((\Delta u)_{i j}\right)^{2} \psi_{R}^{4 m-2}\right) d x\right)^{\frac{1}{2}} .
\end{aligned}
$$


Integrating by parts and using Young's inequality, we obtain

$$
\begin{aligned}
& \int_{A_{\alpha R}^{\beta R}}\left[R^{-2}|\nabla(\Delta u)|^{2} \psi_{R}^{4 m-2}+\left((\Delta u)_{i j}\right)^{2} \psi_{R}^{4 m-2}\right] d x \\
& \leq \int_{B_{\beta R}}\left[R^{-2}|\nabla(\Delta u)|^{2} \psi_{R}^{4 m-2}+\left((\Delta u)_{i j}\right)^{2} \psi_{R}^{4 m-2}\right] d x \\
& =\int_{B_{\beta R}}\left(\Delta^{2} u\right)^{2} \psi_{R}^{4 m-2} d x+\int_{B_{\beta R}} \Delta^{2} u \nabla(\Delta u) \nabla\left(\psi_{R}^{4 m-2}\right) d x \\
& \quad+\int_{B_{\beta R}}|\nabla(\Delta u)|^{2}\left[R^{-2} \psi_{R}^{4 m-2}+\frac{1}{2} \Delta\left(\psi_{R}^{4 m-2}\right)\right] d x \\
& \leq C_{m} \int_{B_{\beta R}}\left(\Delta^{2} u\right)^{2} \psi_{R}^{2 m} d x+C_{m} R^{-2} \int_{B_{\beta R}}|\nabla(\Delta u)|^{2} \psi_{R}^{2 m-2} d x .
\end{aligned}
$$

From (3.31) and (3.32), we obtain

$$
\begin{aligned}
& \left|\int_{B_{\beta R}} \Delta^{2} u \nabla(|x, \nabla(\Delta u)|) \nabla \psi_{R}^{2 m} d x\right| \\
& \quad \leq C\left(\int_{A_{\alpha R}^{\beta R}}\left(\Delta^{2} u\right)^{2} d x\right)^{\frac{1}{2}}\left(\int_{B_{\beta R}}\left(\left(\Delta^{2} u\right)^{2} \psi_{R}^{2 m}+R^{-2}|\nabla(\Delta u)|^{2} \psi_{R}^{2 m-2}\right) d x\right)^{\frac{1}{2}} .
\end{aligned}
$$

The sixth term on the right hand side of (3.18) yields

$$
\begin{aligned}
& \left|\int_{B_{\beta R}} \Delta^{2} u\left(\Delta\left(\psi_{R}^{2 m}\right) \Delta(\langle x, \nabla u\rangle)+2 \nabla\left(\Delta\left(\psi_{R}^{2 m}\right)\right) \nabla(\langle x, \nabla u\rangle)\right) d x\right| \\
& =\mid \int_{B_{\beta R}} \Delta^{2} u\left((x, \nabla(\Delta u)\rangle \Delta\left(\psi_{R}^{2 m}\right)+2 \Delta u \Delta\left(\psi_{R}^{2 m}\right)\right. \\
& \left.\quad+2 \nabla u \nabla\left(\Delta\left(\psi_{R}^{2 m}\right)\right)+2 x_{i} u_{i j}\left(\Delta\left(\psi_{R}^{2 m}\right)\right)_{j}\right) d x \mid \\
& \quad \leq C\left(\int_{A_{\alpha R}^{\beta R}}\left(\Delta^{2} u\right)^{2} d x\right)^{\frac{1}{2}}\left(\int _ { A _ { \alpha R } ^ { \beta R } } \left(R^{-2}|\nabla(\Delta u)|^{2} \psi_{R}^{2 m-2}+R^{-4}(\Delta u)^{2} \psi_{R}^{2 m-4}\right.\right. \\
& \left.\left.\quad+R^{-6}|\nabla u|^{2} \psi_{R}^{2 m-6}+R^{-4}\left|\nabla^{2} u\right|^{2} \psi_{R}^{4 m-6}\right) d x\right)^{\frac{1}{2}} .
\end{aligned}
$$

The last term on the right hand side of (3.18) can be estimated as

$$
\begin{aligned}
& \int_{B_{\beta R}} \Delta^{2} u \Delta\left(\nabla(\langle x, \nabla u\rangle) \nabla\left(\psi_{R}^{2 m}\right)\right) d x \\
& =\int_{B_{\beta R}} \Delta^{2} u\left(3 \nabla(\Delta u) \nabla\left(\psi_{R}^{2 m}\right)+\nabla u \nabla\left(\Delta\left(\psi_{R}^{2 m}\right)\right)+2 \times \nabla\left(u_{i}\right) \times \nabla\left(\psi_{R}^{2 m}\right)_{i}\right) d x \\
& \quad+\int_{B_{\beta R}} \Delta^{2} u\left(x_{i} \times(\Delta u)_{i j} \times\left(\psi_{R}^{2 m}\right)_{j}+u_{i j} \times\left\{x_{i}\left(\Delta\left(\psi_{R}^{2 m}\right)\right)_{j}+2\left(\psi_{R}^{2 m}\right)_{i j}\right\}\right) d x \\
& \quad+2 \int_{B_{\beta R}} x_{i} \Delta^{2} u \times u_{i j k} \times\left(\psi_{R}^{2 m}\right)_{j k} d x .
\end{aligned}
$$


By Hölder's inequality and Young's inequality, we get

$$
\begin{aligned}
& \left|\int_{B_{\beta R}} \Delta^{2} u \Delta\left(\nabla(\langle x, \nabla u\rangle) \nabla\left(\psi_{R}^{2 m}\right)\right) d x\right| \\
& \leq \quad C\left(\int_{A_{\alpha R}^{\beta R}}\left(\Delta^{2} u\right)^{2} d x\right)^{\frac{1}{2}} \\
& \quad \times\left(\int _ { B _ { \beta R } } \left(R^{-2}|\nabla(\Delta u)|^{2} \psi_{R}^{4 m-2}+R^{-6}|\nabla u|^{2} \psi_{R}^{4 m-6}+(\Delta u)_{i j}^{2} \times \psi_{R}^{4 m-2}\right.\right. \\
& \left.\left.\quad+R^{-4}\left|\nabla^{2} u\right|^{2} \times \psi_{R}^{4 m-6}+R^{-2}\left|\nabla^{3} u\right|^{2} \times \psi_{R}^{4 m-4}\right) d x\right)^{\frac{1}{2}} \\
& \leq \\
& \quad\left(\int_{A_{\alpha R}^{\beta R}}\left(\Delta^{2} u\right)^{2} d x\right)^{\frac{1}{2}}\left(\int _ { B _ { \beta R } } \left(\left(\Delta^{2} u\right)^{2} \psi_{R}^{2 m}+\mathbf{B}\left(u, \psi_{R}, m\right)\right.\right. \\
& \left.\left.\quad+R^{-2}\left|\nabla^{3} u\right|^{2} \psi_{R}^{2 m-2}\right) d x\right)^{\frac{1}{2}} .
\end{aligned}
$$

From hypothesis $H_{1}$, one has $(\theta+1) F(s) \leq f(s) s, \forall s \in \mathbb{R}$. Using the latter inequality, (3.25) and $1<\theta<p_{s}(n, 4)$, we get

$$
\int_{B_{\beta R}} F(u)\left\langle\nabla \psi_{R}^{2 m}, x\right\rangle d x=o(1) \quad \text { as } R \rightarrow+\infty .
$$

From (3.18), (3.25)-(3.36), and $1<\theta<p_{s}(n, 4)$, we obtain

$$
\int_{\mathbb{R}^{n}}\left(\Delta^{2} u\right)^{2} d x=\frac{2 n}{n-8} \int_{\mathbb{R}^{n}} F(u) d x .
$$

Now, multiplying equation (1.1) by $u \psi_{R}^{2 m}$ and integrating by parts, we get

$$
\begin{aligned}
\int_{B_{2 R}} & \left(\left(\Delta^{2} u\right)^{2} \psi_{R}^{2 m}-f(u) u \psi_{R}^{2 m}\right) d x \\
= & -\int_{B_{2 R}} \Delta^{2} u\left(2 \Delta u \Delta\left(\psi_{R}^{2 m}\right)+4 u_{i j}\left(\psi_{R}^{2 m}\right)_{i j}+4 \nabla(\Delta u) \nabla\left(\psi_{R}^{2 m}\right)\right. \\
& \left.+4 \nabla u \nabla\left(\Delta\left(\psi_{R}^{2} m\right)\right)+u \Delta^{2}\left(\psi_{R}^{2 m}\right)\right) d x
\end{aligned}
$$

By the same reasoning as above, we find

$$
\int_{\mathbb{R}^{n}}\left(\Delta^{2} u\right)^{2} d x=\int_{\mathbb{R}^{n}} f(u) u d x<\infty .
$$

\section{Proof of Theorems 2.1, 2.2, 2.3, 2.4 and 2.5}

Proof of Theorem 2.1 The proof of Theorem 2.1 for the case $k=1,2,3$ is exactly the same as in $[5,6,8]$. Now, we prove the case $k=4$. Let $u$ be a stable solution to (1.1).

Subcritical case: $1<\theta<p_{s}(n, 4)$. By Proposition 3.1, there exists $C>0$ such that

$$
\int_{B_{R}}|u|^{\theta+1} d x \leq C R^{n-8 \frac{\theta+1}{\theta-1}}, \quad \forall R>0 .
$$


Note that

$$
n-8 \frac{\theta+1}{\theta-1}=n-8-\frac{16}{\theta-1}<0, \quad \forall \theta \in\left(1, p_{s}(n, 4)\right) .
$$

Then, if $1<\theta<p_{s}(n, 4)$, after sending $R \rightarrow \infty$, we get $u \equiv 0$ in $\mathbb{R}^{n}$.

Critical case: $\theta=\frac{n+8}{n-8}$. By Proposition 3.1, we have

$$
\int_{\mathbb{R}^{n}}\left(\left(\Delta^{2} u\right)^{2}+|u|^{\theta+1}\right) d x<+\infty
$$

So,

$$
\lim _{R \rightarrow+\infty} \int_{A_{R}}\left(\left(\Delta^{2} u\right)^{2}+|u|^{\theta+1}\right) d x=0 .
$$

Moreover, if we come back to the proof of Proposition 3.1, we may improve the following integral estimates:

$$
\int_{B_{R}}\left(\left(\Delta^{2} u\right)^{2}+|u|^{\theta+1}\right) d x \leq C \int_{A_{R}}\left(\Delta^{2} u\right)^{2} d x+C R^{-8} \int_{A_{R}} u^{2} d x .
$$

By Hölder's inequality, we have

$$
\begin{aligned}
\int_{B_{R}}\left(\left(\Delta^{2} u\right)^{2}+|u|^{\theta+1}\right) d x & \leq C \int_{A_{R}}\left(\Delta^{2} u\right)^{2} d x+C R^{n \frac{\theta-1}{\theta+1}-8} \times\left(\int_{A_{R}}|u|^{\theta+1} d x\right)^{\frac{2}{\theta+1}} \\
& \leq C \int_{A_{R}}\left(\Delta^{2} u\right)^{2} d x+C\left(\int_{A_{R}}|u|^{\theta+1} d x\right)^{\frac{2}{\theta+1}}
\end{aligned}
$$

Using (4.1), we get

$$
\int_{\mathbb{R}^{n}}|u|^{\theta+1} d x=0
$$

This implies that $u \equiv 0$ in $\mathbb{R}^{n}$.

Proof of Theorem 2.2 We now collect (3.19) and (3.20). By assumption $\mathrm{H}_{3}$, if $u$ is not identically zero, then

$$
\begin{aligned}
\int_{\mathbb{R}^{n}}\left|D^{k} u\right|^{2} d x & =\frac{2 n}{n-2 k} \int_{\mathbb{R}^{n}} F(u) d x \geq\left(1+\alpha_{0}\right) \int_{\mathbb{R}^{n}} f(u) u d x \\
& >\int_{\mathbb{R}^{n}} f(u) u d x=\int_{\mathbb{R}^{n}}\left|D^{k} u\right|^{2} d x .
\end{aligned}
$$

This is a contradiction. Then $u \equiv 0$. The proof of Theorem 2.2 is thus completed.

Proof of Theorem 2.3 The proof of Theorem 2.3 is similar to proof of Proposition 4 in [6]. Let $\gamma \in[1,2 \theta-1+2 \sqrt{\theta(\theta-1)})$. Multiply equation (1.1) by $|u|^{\gamma-1} u \varphi_{R}^{2}$ and integrate by parts to find

$$
\begin{aligned}
& \int_{B_{2 R}} f(u) u|u|^{\gamma-1} \varphi_{R}^{2} d x \\
& \quad=\frac{4 \gamma}{(\gamma+1)^{2}} \int_{B_{2 R}}\left|\nabla\left(|u|^{\frac{\gamma-1}{2}} u\right)\right|^{2} \varphi_{R}^{2} d x-\frac{1}{\gamma+1} \int_{B_{2 R}}|u|^{\gamma+1} \Delta\left(\varphi_{R}^{2}\right) d x .
\end{aligned}
$$


Harrabi et al. Journal of Inequalities and Applications ( 2017) 2017:79

Page 18 of 21

The function $|u|^{\frac{\gamma-1}{2}} u \varphi_{R} \in C_{c}^{1}\left(\mathbb{R}^{n}\right)$, and thus it can be used as a test function in the quadratic form $Q_{u}$. Hence, the stability assumption on $u$ gives

$$
\begin{aligned}
& \int_{B_{2 R}} f^{\prime}(u)|u|^{\gamma+1} \varphi_{R}^{2} d x \\
& \quad \leq \int_{B_{2 R}}\left|\nabla\left(|u|^{\frac{\gamma-1}{2}} u\right)\right|^{2} \varphi_{R}^{2} d x+\int_{B_{2 R}}|u|^{\gamma+1}\left|\nabla \varphi_{R}\right|^{2} d x-\frac{1}{2} \int_{B_{2 R}}|u|^{\gamma+1} \Delta\left(\varphi_{R}^{2}\right) d x .
\end{aligned}
$$

Using (4.2) in the latter, we obtain

$$
\begin{aligned}
& \int_{B_{2 R}}\left\{\left(f^{\prime}(u) u^{2}-\theta f(u) u\right)|u|^{\gamma-1}+\left(\frac{4 \gamma \theta}{(\gamma+1)^{2}}-1\right)\left|\nabla\left(|u|^{\frac{\gamma-1}{2}} u\right)\right|^{2}\right\} \varphi_{R}^{2} d x \\
& \leq C_{1}(\gamma, \theta) \int_{B_{2 R}}|u|^{\gamma+1} \Delta\left(\varphi_{R}^{2}\right) d x+\int_{B_{2 R}}|u|^{\gamma+1}\left|\nabla \varphi_{R}\right|^{2} d x
\end{aligned}
$$

where $C_{1}(\gamma, \theta)=\left(\frac{\theta}{\gamma+1}-\frac{1}{2}\right)$. By hypothesis $H_{1}$, we obtain

$$
\begin{aligned}
& \left(\frac{4 \gamma \theta}{(\gamma+1)^{2}}-1\right) \int_{B_{2 R}}\left|\nabla\left(|u|^{\frac{\gamma-1}{2}} u\right)\right|^{2} \varphi_{R}^{2} d x \\
& \quad \leq C_{1}(\gamma, \theta) \int_{B_{2 R}}|u|^{\gamma+1} \Delta\left(\varphi_{R}^{2}\right) d x+\int_{B_{2 R}}|u|^{\gamma+1}\left|\nabla \varphi_{R}\right|^{2} d x .
\end{aligned}
$$

Since $\theta>1$ and $\gamma \in[1,2 \theta-1+2 \sqrt{\theta(\theta-1)})$, we have $\frac{4 \gamma \theta}{(\gamma+1)^{2}}-1>0$ and

$$
\int_{B_{2 R}}\left|\nabla\left(|u|^{\frac{\gamma-1}{2}} u\right)\right|^{2} \varphi_{R}^{2} d x \leq C(\gamma, \theta) \int_{B_{2 R}}|u|^{\gamma+1}\left(\left|\Delta\left(\varphi_{R}^{2}\right)\right|+\left|\nabla \varphi_{R}\right|^{2}\right) d x
$$

Using again (4.2), we get

$$
\int_{B_{2 R}} f(u) u|u|^{\gamma-1} \varphi_{R}^{2} d x \leq C^{\prime}(\gamma, \theta) \int_{B_{2 R}}|u|^{\gamma+1}\left(\left|\nabla \varphi_{R}\right|^{2}+\left|\Delta\left(\varphi_{R}^{2}\right)\right|\right) d x .
$$

First, we replace $\varphi_{R}$ by $\varphi_{R}^{m}$ in the latter inequality, for any $m>2$, we derive

$$
\begin{aligned}
\int_{B_{2 R}} f(u) u|u|^{\gamma-1} \varphi_{R}^{2 m} d x & \leq C(\gamma, \theta, m) \int_{B_{2 R}}|u|^{\gamma+1} \varphi_{R}^{2 m-2}\left(\left|\nabla \varphi_{R}\right|^{2}+\left|\Delta \varphi_{R}\right|\right) d x \\
& \leq \frac{C}{R^{2}} \int_{B_{2 R}}|u|^{\gamma+1} \varphi_{R}^{2 m-2} d x .
\end{aligned}
$$

By $H_{1}$ and $H_{2}$, we get

$$
\int_{B_{2 R}}|u|^{\theta+\gamma} \varphi_{R}^{2 m} d x \leq \frac{C}{R^{2}} \int_{B_{2 R}}|u|^{\gamma+1} \varphi_{R}^{2 m-2} d x
$$

An application of Young's inequality yields

$$
\int_{B_{2 R}}|u|^{\theta+\gamma} \varphi_{R}^{2 m} d x \leq C R^{n-2 \frac{\theta+\gamma}{\theta-1}}+\frac{\gamma+1}{\gamma+\theta} \int_{B_{2 R}}|u|^{\gamma+\theta} \varphi_{R}^{(2 m-2) \frac{\gamma+\theta}{\gamma+1}} d x .
$$


Thus

$$
\int_{B_{R}}|u|^{\theta+\gamma} d x \leq C^{\prime} R^{n-2 \frac{\theta+\gamma}{\theta-1}} .
$$

As in Farina's work we readily deduce, by letting $R \rightarrow+\infty$, that there is no nontrivial stable solution of (1.1), in the special case $1<\theta<p_{c}(n)$.

Proof of Theorem 2.4 We proceed as in the proof of Proposition 2.1. From (3.11) and (3.13), we deduce by replacing $f(u)$ by $-m u+\lambda|u|^{\theta-1} u-\mu|u|^{p-1} u$ that

$$
\begin{aligned}
& (1-\epsilon) \int_{B_{2 R}}\left(\Delta^{2} u\right)^{2} \varphi_{R}^{2 m} d x-\int_{B_{2 R}}\left(-m u^{2}+\lambda|u|^{\theta+1}-\mu|u|^{p+1}\right) \varphi_{R}^{2 m} d x \\
& \leq C_{\epsilon} R^{-8} \int_{B_{2 R}} u^{2} \varphi_{R}^{2 m-8} d x
\end{aligned}
$$

and

$$
\begin{aligned}
& \int_{B_{2 R}}\left(-m u^{2}+\theta \lambda|u|^{\theta+1}-p \mu|u|^{p+1}\right) \varphi_{R}^{2 m} d x \\
& \quad \leq(1+\epsilon) \int_{B_{2 R}}\left(\varphi_{R}^{m} \Delta^{2} u\right)^{2}+C_{\epsilon} R^{-8} \int_{B_{2 R}} u^{2} \varphi_{R}^{2 m-8} d x .
\end{aligned}
$$

Multiplying (4.3) by $\theta$ and combining it with (4.4), we derive

$$
\begin{aligned}
m(\theta-1) & \int_{B_{2 R}} u^{2} \varphi_{R}^{2 m} d x+\mu(\theta-p) \int_{B_{2 R}}|u|^{p+1} \varphi_{R}^{2 m} d x \\
& +[\theta(1-\epsilon)-(1+\epsilon)] \int_{B_{2 R}}\left(\Delta^{2} u\right)^{2} \varphi_{R}^{2 m} d x \\
\leq & C R^{-8} \int_{B_{2 R}} u^{2} \varphi_{R}^{2 m-8} d x .
\end{aligned}
$$

For $\epsilon$ sufficiently small, we deduce

$$
\begin{aligned}
& m(\theta-1) \int_{B_{2 R}} u^{2} \varphi_{R}^{2 m} d x+\mu(\theta-p) \int_{B_{2 R}}|u|^{p+1} \varphi_{R}^{2 m} d x+\int_{B_{2 R}}\left(\Delta^{2} u\right)^{2} \varphi_{R}^{2 m} d x \\
& \quad \leq C R^{-8} \int_{B_{2 R}} u^{2} \varphi_{R}^{2 m-8} d x .
\end{aligned}
$$

Proof of 1 . If $m>0$ and $\theta \geq p$, then from (4.5), we deduce that

$$
\int_{B_{R}} u^{2} d x \leq C R^{-8} \int_{B_{2 R}} u^{2} d x .
$$

Let $J(R):=\int_{B_{R}} u^{2} d x$. If we iterate the above inequality, then we get

$$
J(R) \leq C R^{-8(k+1)} J\left(2^{k+1} R\right) .
$$

We deduce from the boundedness of $u$ that the right hand side of (4.6) is of order $R^{M}$ with $M=-8(k+1)+n \rightarrow 0$ as $k \rightarrow+\infty$. Hence, we can choose $k$ large enough such that $M<0$. 
Then it follows from (4.6) that $J(R) \rightarrow 0$, as $R \rightarrow+\infty$. So we get

$$
\int_{\mathbb{R}^{n}} u^{2} d x=0
$$

Then $u \equiv 0$.

Proof of 2. If $\theta>p, m \geq 0$, then from (4.5) and by Young's inequality, we get

$$
\int_{B_{2 R}}|u|^{p+1} \varphi_{R}^{2 m} d x+\int_{B_{2 R}}\left(\Delta^{2} u\right)^{2} \varphi_{R}^{2 m} d x \leq \frac{2}{p+1} \int_{B_{2 R}}|u|^{p+1} \varphi_{R}^{(p+1)(m-4)} d x+C R^{n-8 \frac{p+1}{p-1}} .
$$

Choosing $2 m=(p+1)(m-4)$, thus

$$
\int_{B_{2 R}}|u|^{p+1} \varphi_{R}^{2 m} d x+\int_{B_{2 R}}\left(\Delta^{2} u\right)^{2} \varphi_{R}^{2 m} d x \leq C R^{n-8 \frac{p+1}{p-1}}
$$

Consequently

$$
\int_{B_{R}}|u|^{p+1} d x+\int_{B_{R}}\left(\Delta^{2} u\right)^{2} d x \leq C R^{n-8 \frac{p+1}{p-1}}
$$

The result then follows in a similar way to that in the proof of Theorem 2.1. This completes the proof of Theorem 2.4.

Proof of Theorem 2.5 We can proceed as in the proof of Proposition 3.4, we get

$$
\int_{\mathbb{R}^{n}}\left|D^{k} u\right|^{2}=\frac{2 n}{n-2 k} \int_{\mathbb{R}^{n}}\left(-\frac{m}{2} u^{2}+\frac{\lambda}{\theta+1}|u|^{\theta+1}-\frac{\mu}{p+1}|u|^{p+1}\right)
$$

and

$$
\int_{\mathbb{R}^{n}}\left|D^{k} u\right|^{2}=\int_{\mathbb{R}^{n}}\left(-m u^{2}+\lambda|u|^{\theta+1}-\mu|u|^{p+1}\right) .
$$

Thus

$$
\begin{aligned}
& \frac{2 m k}{n-2 k} \int_{\mathbb{R}^{n}} u^{2} d x+\lambda\left(1-\frac{2 n}{(n-2 k)(\theta+1)}\right) \int_{\mathbb{R}^{n}}|u|^{\theta+1} d x \\
& +\mu\left(\frac{2 n}{(n-2 k)(p+1)}-1\right) \int_{\mathbb{R}^{n}}|u|^{p+1} d x=0 .
\end{aligned}
$$

This concludes the proof of Theorem 2.5 .

Competing interests

The authors declare that they have no competing interests.

Authors' contributions

All authors contributed equally to the writing of this paper. All authors read and approved the final manuscript.

\section{Author details}

'Institut Supérieur des Mathématiques Appliquées et de I'Informatique de Kairouan, Université de Kairouan, Kairouan,

Tunisia. ${ }^{2}$ Faculté des Sciences, Département de Mathématiques, Université de Sfax, B.P. 1171, 3000, Sfax, Tunisia. 


\section{Acknowledgements}

The authors wish to thank Professor D. Ye for stimulating discussions on the subject. Also, A. Harrabi, B. Rahal and M.K. Hamdani would like to express their deepest gratitude to our Research Laboratory LR11ES53 Algebra, Geometry and Spectral Theory (AGST) Sfax University, for providing us with an excellent atmosphere for doing this work.

\section{Publisher's Note}

Springer Nature remains neutral with regard to jurisdictional claims in published maps and institutional affiliations.

Received: 1 September 2016 Accepted: 31 March 2017 Published online: 18 April 2017

\section{References}

1. Berestycki, H, Lions, PL: Nonlinear scalar field equations, I, Existence of ground state, II, Existence of infinitely many solutions. Arch. Ration. Mech. Anal. 82, 313-345 (1983)

2. Bahri, A, Lions, PL: Solutions of superlinear elliptic equations and their Morse indices. Commun. Pure Appl. Math. 45, 1205-1215 (1992)

3. Cowan, C, Ghoussoub, N: Regularity of semi-stable solutions to fourth order nonlinear eigevalue problems on general domains (2012). arXiv:1206.3471v1

4. Dancer, EN: Superlinear problems on domains with holes of asymptotic shape and exterior problems. Math. Z. 229(3), 475-491 (1998)

5. Dávila, J, Dupaigne, L, Wang, K, Wei, J: A monotonicity formula and a Liouville-type theorem for a fourth order supercritical problem. Adv. Math. 258, 240-285 (2014)

6. Farina, A: On the classification of solutions of the Lane-Emden equation on unbounded domains of $\mathbb{R}^{n}$. J. Math. Pures Appl. 87, 537-561 (2007)

7. Hajlaoui, H, Harrabi, A, Ye, D: On stable solutions of biharmonic problem with polynomial growth. Pac. J. Math. 270, 79-93 (2014)

8. Harrabi, A, Rahal, B: On the sixth-order Joseph-Lundgren exponent. Ann. Henri Poincaré 18(3), 1055-1094 (2017). doi:10.1007/s00023-016-0522-5

9. Harrabi, A, Rebhi, S, Selmi, S: Solutions of superlinear equations and their Morse indices I. Duke Math. J. 94, 141-157 (1998)

10. Harrabi, A, Rebhi, S, Selmi, S: Solutions of superlinear equations and their Morse indices II. Duke Math. J. 94, 159-179 (1998)

11. Wei, J, Ye, D: Liouville theorems for stable solutions of biharmonic problem. Math. Ann. 356(4), 1599-1612 (2013)

12. Wei, J, Xu, X, Yang, W: On the classification of stable solutions to biharmonic problems in large dimensions. Pac. J. Math. 263(2), 495-512 (2013)

13. Dupaigne, L, Farina, A: Stable solutions of $-\Delta u=f(u)$ in $\mathbb{R}^{n}$. J. Eur. Math. Soc. 12, 855-882 (2010)

14. Ramos, N, Rodrigues, P: On a fourth order superlinear elliptic problem. Electron. J. Differ. Equ., Conf. 6, 243-255 (2001)

15. Souplet, P: The proof of the Lane-Emden conjecture in four space dimensions. Adv. Math. 221, 1409-1427 (2009)

16. Cowan, C, Esposito, P, Ghoussoub, N: Regularity of extremal solutions in fourth order nonlinear eigenvalue problems on general domains. Discrete Contin. Dyn. Syst., Ser. A 28, 1033-1050 (2010)

17. Dupaigne, L, Ghergu, M, Goubet, O, Warnault, G: Entire large solutions for semilinear elliptic equations. J. Differ. Equ. 253, 2224-2251 (2012)

18. Gazzola, F, Grunau, HC: Radial entire solutions for supercritical biharmonic equations. Math. Ann. 334, 905-936 (2006)

19. Blatt, S: Monotonicity formulas for extrinsic triharmonic maps and the triharmonic Lane-Emden equation. J. Differ. Equ. (2017). doi:10.1016/j.jde.2017.01.025

20. Hajlaoui, H, Harrabi, A, Mtiri, F: Morse indices of solutions for super-linear elliptic PDEs. Nonlinear Anal. 116, 180-192 (2015)

21. Wei, J, Xu, X: Classification of solutions of high order conformally invariant equations. Math. Ann. 313(2), 207-228 (1999)

22. Kavian, O: Intoduction à la théorie des points critiques. In: Mathmatiques et Applications, vol. 13. Springer, Berlin (1991)

23. Gidas, B, Spruck, J: A priori bounds for positive solutions of nonlinear elliptic equations. Commun. Partial Differ. Equ. 6 883-901 (1981)

\section{Submit your manuscript to a SpringerOpen ${ }^{\ominus}$ journal and benefit from:}

- Convenient online submission

- Rigorous peer review

- Immediate publication on acceptance

- Open access: articles freely available online

- High visibility within the field

- Retaining the copyright to your article 